\title{
sFGFR for achondroplasia
}

\section{By Lauren Martz, Staff Writer}

Achondroplasia treatments include surgical procedures to increase bone length and human growth hormone injections to stimulate growth, but neither restores normal stature. Moreover, additional interventions are required to treat the secondary consequences of short-limb dwarfism including paralysis, respiratory disorders, sleep apnea and spinal deformities.

Now, French researchers have designed a decoy version of fibroblast

\section{"Our treatment was} effective at both restoring the stature of treated mice and preventing complications. This was critical for the treatment to have benefits over existing approaches."

-Elvire Gouze, Institut National de la Santé et de la Recherche Médicale increasing FGFR3 signaling.

The increased signaling ultimately inhibits normal chondrocyte function and maturation, impairing normal bone growth.

Achondroplasia research has turned toward correcting the abnormal FGFR3 signaling in children. Last year, one such treatment from BioMarin Pharmaceutical Inc. began clinical testing.

The company's BMN-111 is an analog of C-type natriuretic peptide (CNP; NPPC) that blocks signaling downstream of FGFR3 in the growth plate. The molecule has completed a Phase I trial in healthy adults, and BioMarin hopes to begin a Phase II trial in patients this year or in 1Q14.

In animal models, BMN-111 was safe and restored bone growth during the growth period, resulting in bones of normal length. The compound has a half-life of 45 minutes, which VP of Pharmacological Sciences Charles O’Neill said may require daily dosing.

Now, Elvire Gouze and colleagues at Institut National de la Santé et de la Recherche Médicale U1065 (INSERM U1065) have taken a different approach to blocking aberrant FGFR3 signaling in achondroplasia. The team designed a soluble human FGFR3 (sFGFR3) receptor that acts as a decoy for FGFR3 ligands to decrease ligand binding and receptor signaling.

Gouze is a researcher at INSERM U1065. The paper also included researchers from the University of Paul Sabatier Toulouse III, the University of Nice Sophia Antipolis, University Hospital Center of l'Archet and Institute National de la Santé et de la Recherche Médicale U1043 (INSERM U1043).

In a mouse model of achondroplasia, the team subcutaneously injected 0.25 or $2.5 \mathrm{mg} / \mathrm{kg}$ of sFGFR3 twice weekly for three weeks in newborn mice.

The animals had increased skeletal growth and long-bone length and decreased mortality compared with vehicle-treated controls. Bone length in treated mice was comparable to that in healthy controls.

sFGFR3 also penetrated the cartilage matrix of the growth plate, stimulated chondrocyte maturation and increased synthesis of extracellular matrix components. These findings suggest that sFGFR3 restores chondrocyte maturation blocked by the aberrant sFGFR3 activation in achondroplasia.

The next question was whether sFGFR3 could correct achondroplasia's effects on spinal and skull abnormalities. Whereas $80 \%$ of vehicle-treated mice had spinal deformities, only $12 \%$ and $6 \%$ of mice receiving low and high sFGFR3 doses, respectively, developed abnormalities.

sFGFR3 also corrected skull length. The molecule was safe and had a half-life of about 16 hours in mice.

Data were published in Science Translational Medicine.

"Our treatment was effective at both restoring the stature of treated mice and preventing complications. This was critical for the treatment to have benefits over existing approaches," said Gouze.

"This strategy may also work as a treatment for very severe complications that occur due to FGFR3 mutations, such as severe achondroplasia with developmental delay and acanthosis nigricans, which is characterized by extremely short stature and intellectual disability," said Kalina Hristova, professor of materials science and biomedical engineering at The Johns Hopkins University.

\section{Halftime}

Gouze said that her team needs to verify that sFGFR3's half-life in humans is at least equivalent to that in mice.

She added, "We cannot plan a dosing regimen in humans as we need to verify the pharmacokinetic parameters in humans first. We can just say that it would certainly not be daily injections."

O'Neill acknowledged that sFGFR3 has a longer half-life and the potential for less frequent dosing. However, he noted that "this could be a good and a bad thing. If any toxicities do arise from treatment with SFGFR3, it would take longer to resolve the toxicological response. In comparison, we have found that normal bone growth patterns are restored within one week of cessation of treatment with BMN-111."

O'Neill also said that decoy receptors "have the potential to cause immunogenicity issues, especially when tested in higher species. Antibodies against the protein could decrease the therapeutic benefit and could also cause toxicity issues if there is any cross-reactivity for related human receptors." 


\section{ANALYSIS}

\section{TARGETS \& MECHANISMS}

He wanted to see mice dosed with sFGFR3 for longer periods of time to characterize any immunogenicity and cross-reactivity for endogenous FGFRs.

O'Neill said that BioMarin has seen a weak immunological response to BMN-111 in animal studies, but the presence of antibodies did not affect the pharmacological activity of the drug or the safety profile. BMN-111 is 39 amino acids long, whereas sFGFR3 is about 700 amino acids long.

Hristova added that the use of a full-length protein could also be associated with high costs.

"The authors use the full-length extracellular domain of FGFR3 and produce it in mammalian cells. This treatment may be too expensive, especially if long-term therapy is required. It should be investigated if the post-translational modifications of the soluble FGFR3 are required for this application. If they are not, it may be possible to produce the protein in bacteria in a cost-effective way," she said.

Finally, O'Neill noted the large amount of sFGFR3 needed for therapeutic activity. "The molecular characteristics of sFGFR3 don't favor movement to the growth plate," which is a cartilage component at the end of bones in children and adolescents that is involved in initial development and growth. "This could be the reason that high doses are required for therapeutic effect in the mice, and the researchers may need to tweak the molecule to allow easier distribution to the growth plate."

Gouze told SciBX that INSERM U1065 has filed an international patent application and that the IP is available for licensing.

Martz, L. SciBX 6(40); doi:10.1038/scibx.2013.1120

Published online Oct. 17, 2013

\section{REFERENCES}

1. Garcia, S. et al. Sci. Transl. Med.; published online Sept. 13, 2013; doi:10.1126/scitranslmed.3006247

Contact: Elvire Gouze, Institut National de la Santé et de la Recherche Médicale (INSERM), Nice, France e-mail: elvire.gouze@inserm.fr

\section{COMPANIES AND INSTITUTIONS MENTIONED}

BioMarin Pharmaceutical Inc. (NASDAQ:BMRN), Novato, Calif. Institut National de la Santé et de la Recherche Médicale U1043, Toulouse, France

Institut National de la Santé et de la Recherche Médicale U1065, Nice, France

The Johns Hopkins University, Baltimore, Md.

University Hospital Center of l'Archet, Nice, France

University of Nice Sophia Antipolis, Nice, France

University of Paul Sabatier Toulouse III, Toulouse, France 